\title{
Vortex reconnections in classical and quantum fluids
}

\author{
Alberto Enciso $^{1} \cdot$ Daniel Peralta-Salas $^{1}$ D \\ Received: 11 June 2021 / Accepted: 6 November 2021 / Published online: 24 November 2021 \\ (c) The Author(s) 2021
}

\begin{abstract}
We review recent rigorous results on the phenomenon of vortex reconnection in classical and quantum fluids. In the context of the Navier-Stokes equations in $\mathbb{T}^{3}$ we show the existence of global smooth solutions that exhibit creation and destruction of vortex lines of arbitrarily complicated topologies. Concerning quantum fluids, we prove that for any initial and final configurations of quantum vortices, and any way of transforming one into the other, there is an initial condition whose associated solution to the Gross-Pitaevskii equation realizes this specific vortex reconnection scenario. Key to prove these results is an inverse localization principle for Beltrami fields and a global approximation theorem for the linear Schrödinger equation.
\end{abstract}

Keywords Navier-Stokes equations · Gross-Pitaevskii equation · Vortex · Reconnection · Global approximation theorem $\cdot$ Stability theorem

Mathematics Subject Classification 35Q30 · 35Q55

\section{Introduction}

In this section we present the equations that model classical and quantum fluids. We also introduce the concept of vortex (whose definition depends on the context) and the important phenomenon of vortex reconnection, which is related to the creation or destruction of topological structures during the vortex dynamics. A different area of physics where reconnections play a major role is in magnetohydrodynamics (MHD), where one talks about magnetic reconnections, but we shall not deal with this topic here.

\footnotetext{
$\bowtie \quad$ Daniel Peralta-Salas

dperalta@icmat.es

Alberto Enciso

aenciso@icmat.es

1 Instituto de Ciencias Matemáticas, Consejo Superior de Investigaciones Científicas, 28049 Madrid, Spain
} 


\subsection{Classical fluids: the Navier-Stokes equations}

The dynamics of an incompressible fluid flow with viscosity is described by the Navier-Stokes equations,

$$
\partial_{t} u+(u \cdot \nabla) u-v \Delta u=-\nabla P, \quad \operatorname{Div} u=0, \quad u(\cdot, 0)=u_{0},
$$

where the unknowns are the velocity field of the fluid $u(x, t)$ and the hydrodynamic pressure $P(x, t)$. Here the viscosity $v>0$ is a fixed constant. Usually, the spatial variable takes values in $\mathbb{R}^{3}$ or $\mathbb{R}^{3}$ with periodic boundary conditions, i.e., the torus $\mathbb{T}^{3}:=\mathbb{R}^{3} /(2 \pi \mathbb{Z})^{3}$.

A time-dependent vector field that plays a crucial role in fluid mechanics is the vorticity, defined by

$$
\omega:=\operatorname{curl} u \text {. }
$$

The integral curves of the vorticity $\omega(x, t)$ at fixed time $t$, that is to say, the solutions to the autonomous ODE

$$
\dot{x}(\tau)=\omega(x(\tau), t)
$$

for some initial condition $x(0)=x_{0}$, are the vortex lines of the fluid at time $t$.

When the fluid has no viscosity $(v=0)$, the Navier-Stokes equations become the Euler equations. In this case, it is well known that the vorticity is transported by the velocity field (a phenomenon known as Helmholtz's transport of vorticity),

$$
\partial_{t} \omega=(\omega \cdot \nabla) u-(u \cdot \nabla) \omega=:[\omega, u] .
$$

This equation ensures that the vorticity at time $t$ can be written in terms of the initial vorticity $\omega_{0}$ as

$$
\omega(\cdot, t)=\left(\phi_{t}\right)_{*} \omega_{0},
$$

that is, as the push-forward of the initial vorticity along the time $t$ flow $\phi_{t}$ generated by the velocity field. Therefore, the set of vortex lines at any times $t_{0}$ and $t_{1}$ are diffeomorphic as long as the solution remains smooth on the time interval $\left[t_{0}, t_{1}\right]$. In short, the vortex lines do not change their topology.

However, in the presence of viscosity, the vorticity is no longer transported along the flow, and the diffusive term gives rise to a different phenomenon known as vortex reconnection. Briefly, one says that a vortex reconnection has occurred at time $T$ if the vortex lines at time $T$ and at time 0 are not homeomorphic, so there has been a change of topology $[5,10]$. For example, certain vortex lines can break and new vortex lines, possibly knotted or linked in a different way, can be created (see Fig. 1).

There is overwhelming numerical and physical evidence of the fact that vortex reconnection occurs $[5,10,19]$. We highlight the recent experimental results presented in $[11,17]$, where the authors study how vortex lines (or tubes) of different knotted topologies reconnect in actual fluids using cleverly designed hydrofoils. In contrast with the wealth of heuristic, numerical and experimental results on this subject, the first mathematically rigorous scenario of vortex reconnection was constructed very recently in [7] (see Theorem 2.1). 


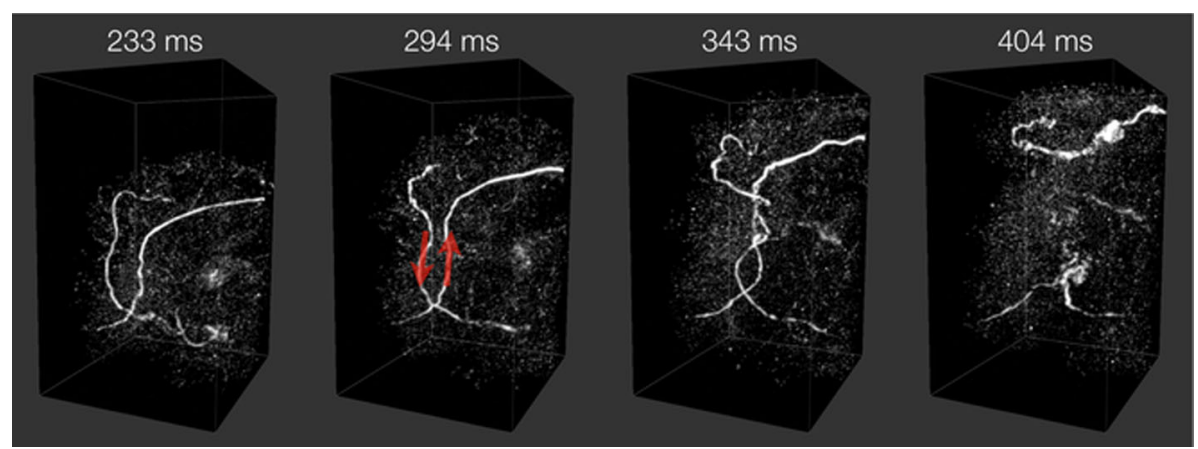

Fig. 1 Reconnection of vortex lines of a fluid flow measured at the Irvine Lab in Chicago (2012). Courtesy of William Irvine [11]

Fig. 2 Visual description of the reconnection phenomenon. Here a quantum vortex in the shape of a trefoil knot reconnects into two linked unknots. Courtesy of William Irvine [12]

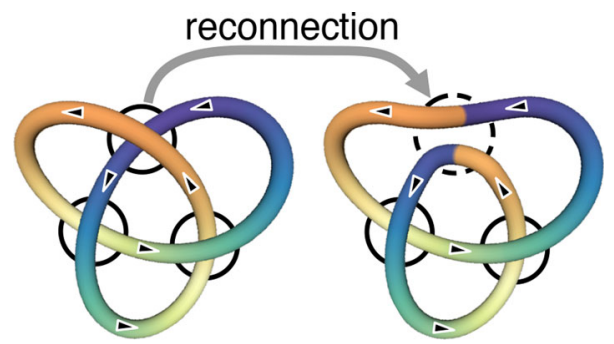

\subsection{Quantum fluids: the Gross-Pitaevskii equation}

A quantum fluid or Bose-Einstein condensate (sometimes also called a superfluid) is described by the Gross-Pitaevskii equation,

$$
i \partial_{t} \psi+\Delta \psi+\left(1-|\psi|^{2}\right) \psi=0, \quad \psi(\cdot, 0)=\psi_{0},
$$

where the unknown is the complex-valued wave function $\psi(x, t)$, with $x \in \mathbb{R}^{3}$.

We are interested in solutions $\psi(x, t)$ that tend to 1 as $|x| \rightarrow \infty$. The reason is the relation between the Gross-Pitaevskii equation and the Ginzburg-Landau functional

$$
\mathcal{E}[\psi](t):=\int_{\mathbb{R}^{3}}\left(\frac{1}{2}|\nabla \psi(x, t)|^{2}+\frac{1}{4}\left(1-|\psi(x, t)|^{2}\right)^{2}\right) d x,
$$

so one picks solutions that tend to 1 fast enough at infinity to have finite Ginzburg-Landau energy.

A very active topic in condensed matter physics is the study of the evolution of quantum vortices [1]. Recall that the quantum vortices of the superfluid at time $t$ are defined as the connected components of the set

$$
Z_{\psi}(t):=\left\{x \in \mathbb{R}^{3}: \psi(x, t)=0\right\},
$$

so, as $\psi$ is complex valued, they are typically given by closed curves in space.

A central aspect is the analysis of the quantum vortex reconnection, that is, the process through which two quantum vortices cross, each of them breaking into two parts and exchanging part of itself for part of the other (see Fig. 2). This may lead to a change of topology of the quantum vortices. Among the extensive literature on this topic, an outstanding contribution 
is the first experimental measurement of vortex reconnection in superfluid helium [3]. In this paper it was observed that the distance between the vortices behaves as $C|t-T|^{1 / 2}$ near the reconnection time $T$. Further numerical [12,18] and theoretical [16] studies have analyzed quantum vortex reconnections in detail, showing that the above separation rate is in fact universal (this is nowadays called the $t^{1 / 2}$ law). Another remarkable numerical observation [12] is that the parity of the number of quantum vortices changes at the reconnection time, i.e., an even number of vortices reconnect into an odd number of quantum vortices and viceversa.

We proved recently [6] that, given any finite initial and final configurations of quantum vortices (which do not need to be topologically equivalent) and any reasonable way of reconnecting them, there is a smooth initial datum $\psi_{0}$ whose associated solution realizes this specific vortex reconnection scenario. Moreover, this solution obeys the aforementioned $t^{1 / 2}$ law and parity switch. See Theorem 2.3 for details.

\section{Statement of the theorems}

In this section we state the main theorems that we proved in [6,7]. The first result shows the existence of smooth solutions to the Navier-Stokes equations in $\mathbb{T}^{3}$ that exhibit a finite cascade of changes of topology of the vorticity field (which implies the existence of vortex reconnections). The second theorem establishes that, given any finite initial and final configurations of quantum vortices and any conceivable way of transforming one into the other, there is a smooth initial datum $\psi_{0}$ whose associated solution to the Gross-Pitaevskii equation in $\mathbb{R}^{3}$ realizes this reconnection scenario.

\subsection{Existence of vortex reconnection in Navier-Stokes}

To state the theorem, let us prescribe the following data:

(i) Finitely many reconnection times: $0=$ : $T_{0}<T_{1}<\cdots<T_{n}$.

(ii) The energy ( $L^{2}$ norm) of the initial condition: $M>0$.

(iii) For each odd integer $k \in[1, n]$ a finite collection $\mathcal{V}_{k}$ of closed curves (pairwise disjoint but possibly knotted and linked) that are contained in a ball of $\mathbb{T}^{3}$.

The following theorem [7] establishes the existence of vortex reconnections for smooth global solutions of the Navier-Stokes equations. In the statement, we say that two sets $\mathcal{K}_{1}$ and $\mathcal{K}_{2}$ of $\mathbb{T}^{3}$ are isotopic if there exists a diffeomorphism $\Phi$ of $\mathbb{T}^{3}$, connected with the identity, such that $\Phi\left(\mathcal{K}_{1}\right)=\mathcal{K}_{2}$.

Theorem 2.1 There is a global smooth solution $u: \mathbb{T}^{3} \times[0, \infty) \rightarrow \mathbb{R}^{3}$ of the Navier-Stokes equations on $\mathbb{T}^{3}$, with an initial datum $u_{0}$ of norm $\left\|u_{0}\right\|_{L^{2}}=M$ and of zero mean, which, for each odd integer $k \in[1, n]$, exhibits at time $T_{k}$ a set of vortex lines isotopic to $\mathcal{V}_{k}$, and when $k \leq n-1$ this set is not isotopic to any subset of the vortex lines of the fluid at time $T_{k-1}$ or $T_{k+1}$.

Remark 2.2 This scenario of vortex reconnection is structurally stable. By this we mean that the vortex reconnection phenomenon occurs for any initial datum that is close enough in the $C^{2}\left(\mathbb{T}^{3}\right)$ norm to the initial velocity $u_{0}$ of the theorem (and therefore the vorticities are $C^{1}$-close so that one can apply the hyperbolic permanence theorem). Moreover, the existence of non-homeomorphic vortex structures occurs not only between the times $T_{k}$ and $T_{k \pm 1}$ with $k$ odd, but also between any nonnegative times $t_{k}$ and $t_{k \pm 1}$ for which $\left|T_{k}-t_{k}\right|+\left|T_{k \pm 1}-t_{k \pm 1}\right|$ is small enough. This ensures that the vortex reconnection is experimentally observable. 
The proof that this scenario of vortex reconnection occurs [7] is indirect, meaning that we prove that there has been a change in the topology of the vortex structures of the fluid but we cannot describe the way in which these structures break. Informally, this theorem does not provide a movie of how the reconnection process happens but only some significant snapshots of it. In particular, we do not know if the vortex reconnection happens essentially as in the well-known model [19] of two rotating columns of fluid that meet at an angle that evolve into a configuration similar to cutting both columns in half at their point of contact and then reconnecting each end of the first column with one of the second.

\subsection{Existence of vortex reconnection in Gross-Pitaevskii}

The initial and final vortex configurations are described by links $\Gamma_{0}, \Gamma_{1} \subset \mathbb{R}^{3}$. We recall that a link is a finite union of smooth closed curves in $\mathbb{R}^{3}$ that are pairwise disjoint. Notice that $\Gamma_{0}$ and $\Gamma_{1}$ do not necessarily have the same number of connected components, and that these components need not be homeomorphic. To describe a way of transforming the link $\Gamma_{0}$ into $\Gamma_{1}$ in time $T$, we introduced in [6] the notion of a pseudo-Seifert surface. By this we mean a smooth, two-dimensional, bounded, orientable surface $\Sigma \subset \mathbb{R}^{4}$ whose boundary is

$$
\partial \Sigma=\left(\Gamma_{0} \times\{0\}\right) \cup\left(\Gamma_{1} \times\{T\}\right) .
$$

We then say that the links $\Gamma_{0}$ and $\Gamma_{1}$ are connected by the pseudo-Seifert surface $\Sigma$. As an additional technical assumption, we will assume that the surface is in generic position, meaning that the time coordinate of $\mathbb{R}^{4}$ is a Morse function on $\Sigma$ that does not have any critical points on the boundary $\partial \Sigma$. This kind of pseudo-Seifert surfaces can be used to describe any reconnection cascade like the ones numerically studied in [12]. In fact, pseudoSeifert surfaces provide a universal mechanism to describe the reconnection process for the initial and final links $\Gamma_{0}, \Gamma_{1}$.

The theorem can then be stated as follows. Given a spacetime subset $\Omega \subset \mathbb{R}^{n+1}$ (here, $n=3$ ), let us denote by

$$
\Omega_{t}:=\Omega \cap\left(\mathbb{R}^{n} \times\{t\}\right)
$$

its intersection with the time $t$ slice. Furthermore, we use the notation

$$
\Lambda_{\eta}(x):=\eta x
$$

for the dilation on $\mathbb{R}^{n}$ with ratio $\eta>0$.

Theorem 2.3 Consider two links $\Gamma_{0}, \Gamma_{1} \subset \mathbb{R}^{3}$ and a pseudo-Seifert surface $\Sigma \subset \mathbb{R}^{4}$ connecting $\Gamma_{0}$ and $\Gamma_{1}$ in time $T>0$. Then, there is a global smooth solution $\psi(x, t)$ to the Gross-Pitaevskii equation on $\mathbb{R}^{3}$, tending to 1 at infinity, which realizes the vortex reconnection pattern described by $\Sigma$ up to a diffeomorphism. Specifically, for any $\varepsilon>0$ and any $k>0$, one has:

(i) The function $\psi$ tends to 1 exponentially fast at infinity. More precisely, $1-\psi \in$ $C_{\mathrm{loc}}^{\infty}\left(\mathbb{R}, \mathcal{S}\left(\mathbb{R}^{3}\right)\right)$, where $\mathcal{S}\left(\mathbb{R}^{3}\right)$ is the Schwartz space.

(ii) One can track the evolution of the quantum vortices during the prescribed reconnection process at all times. More precisely, there is some $\eta>0$ and a diffeomorphism $\Psi$ of $\mathbb{R}^{4}$ with $\|\Psi-\mathrm{id}\|_{C^{k}\left(\mathbb{R}^{4}\right)}<\varepsilon$ such that $\Lambda_{\eta}\left[\Psi(\Sigma)_{t}\right]$ is a union of connected components of $Z_{\psi}\left(\eta^{2} t\right)$ for all $t \in[0, T]$.

(iii) The separation distance obeys the $t^{1 / 2}$ law and the parity of the number of quantum vortices changes at each reconnection time. 
Remark 2.4 The parameter $\eta>0$ is small, which means that the reconnection process takes place in a small space region of size of order $\eta$ during a very short time scale $\eta^{2} T$. After the time scale $\eta^{2} T$, our method of proof does not provide any information of the evolution of the solution $u(x, t)$ of the Gross-Pitaevskii equation.

\subsection{A comparison between both reconnection theorems}

In stark contrast with Theorem 2.1 for the Navier-Stokes equations, in Theorem 2.3 for the Gross-Pitaevskii equation we are able to track the evolution of the quantum vortices during the whole process, which permits to describe the reconnection events in detail and verify that they exhibit the properties observed in the physics literature. The way we describe the process is through pseudo-surfaces in space-time, as explained above. This crucially uses the scalar nature of the problem. Unfortunately, for vectorial equations one cannot hope to find an analogous way of encoding reconnections.

From the point of view of the strategy of the proof, Theorem 2.1 involves two ideas. Firstly, one comes up with a (rather sophisticated) construction of a family of Beltrami fields (that is, eigenfunctions of the curl operator) of arbitrarily high frequency that present vortex lines of "robustly distinct" topologies. This step is time-independent. The time-dependent part of the argument hinges on the idea of transition to lower frequencies: acting in the linear regime, the diffusive part of the equation guarantees that a high-frequency Beltrami field can represent the leading part of the solution at time $T_{0}$ while a Beltrami field of a still high but much lower frequency may dominate at a fixed later time $T_{1}$. It is obvious that this heat-equation-type argument will not work for the Gross-Pitaevskii equation even in the linear regime, which is controlled by the Schrödinger equation. Instead, the proof of Theorem 2.3 relies on a new global approximation property of the linear Schrödinger equation.

\section{Technical tools}

In this section we introduce two tools, of interest themselves, which are instrumental for the proofs of Theorems 2.1 and 2.3. First we recall the concept of Beltrami fields on $\mathbb{T}^{3}$ and show that they satisfy a remarkable inverse localization property [8]. Roughly speaking, this means that any Beltrami field on a bounded domain of $\mathbb{R}^{3}$ can be transplanted, up to a controlled error, into a high-frequency Beltrami field on $\mathbb{T}^{3}$. The second result we present is a new global approximation theorem [6] for the linear Schrodinger equation in $\mathbb{R}^{n}$ that allows one to approximate a local solution defined in a bounded spacetime domain by a global solution of the Schrödinger equation.

\subsection{Inverse localization of Beltrami fields}

A Beltrami field on $\mathbb{T}^{3}$ is a vector field satisfying the equation

$$
\operatorname{curl} v=\lambda v
$$

for some real number $\lambda \neq 0$. It is easy to see that such fields are divergence-free and have zero mean.

It is well-known [7] that the spectrum of the curl operator on $\mathbb{T}^{3}$ consists of the numbers of the form $\lambda= \pm|k|$ for some vector with integer coefficients $k \in \mathbb{Z}^{3}$. For concreteness, we will henceforth assume that $\lambda>0$. 
A famous class of Beltrami fields on the torus are the ABC fields [2], which can be written in terms of three real constants as

$$
v=\left(A \sin x_{3}+C \cos x_{2}, B \sin x_{1}+A \cos x_{3}, C \sin x_{2}+B \cos x_{1}\right) .
$$

They correspond to the lowest positive eigenvalue $\lambda=1$, which has multiplicity 6 .

To state the main result of this subsection, we first introduce some notation. Let us fix an arbitrary point $p_{0} \in \mathbb{T}^{3}$ and take a patch of normal geodesic coordinates $\Psi: \mathbb{B}_{\rho} \rightarrow B_{\rho}$ centered at $p_{0}$. Here $B_{\rho}$ (resp. $\mathbb{B}_{\rho}$ ) denotes the ball in $\mathbb{R}^{3}$ (resp. the geodesic ball in $\mathbb{T}^{3}$ ) centered at the origin (resp. at $p_{0}$ ) and of radius $\rho>0$; we shall drop the subscript when $\rho=1$.

The inverse localization theorem shows that any Beltrami field on a bounded set of $\mathbb{R}^{3}$ can be reproduced in a ball of size $\lambda^{-1}$ by a Beltrami field on $\mathbb{T}^{3}$ with high enough eigenvalue $\lambda$.

Theorem 3.1 Let $w$ be a Beltrami field in $\mathbb{R}^{3}$, satisfying curl $w=w$, and fix any positive real $\varepsilon$ and integer $r$. Then for any large enough odd integer L there is a Beltrami field $v$ on $\mathbb{T}^{3}$, satisfying curl $v=L v$, such that

$$
\left\|w-v \circ \Psi^{-1}\left(\frac{\cdot}{L}\right)\right\|_{C^{r}(B)}<\varepsilon .
$$

\subsection{A global approximation theorem for the Schrödinger equation}

Global approximation theorems are classical in the case of elliptic operators [4,13,14], starting with the work of Runge in complex analysis. Roughly speaking, this theory establishes a sort of flexibility for solutions that satisfy a linear elliptic equation on a bounded domain $U \subset \mathbb{R}^{n}$ : the local solution can be uniformly approximated in compact subsets of $U$ by a global solution to the same equation provided that $\mathbb{R}^{n} \backslash U$ does not have any bounded connected components.

These results have been recently extended to the related setting of parabolic operators [9]. The case of dispersive equations, however, is substantially different and presents new key technical subtleties. The way we followed in [6] to solve these difficulties hinges on a careful analysis of integrals defined by Bessel functions with real and complex arguments. Roughly speaking, we proved that a function that satisfies the Schrödinger equation on a spacetime set with certain mild topological properties can be approximated, in a suitable norm, by a global solution of the form $e^{i t \Delta} \psi_{0}$, with $\psi_{0}$ a Schwartz function.

To state the global approximation theorem for the Schrödinger equation that we proved in [6], we need to introduce some notation. Specifically, we use the norm

$$
\|\chi\|_{L^{2} H^{s}(\Omega)}^{2}:=\int_{-\infty}^{\infty}\|\chi(\cdot, t)\|_{H^{s}\left(\Omega_{t}\right)}^{2} d t<\infty,
$$

where $s$ is any real number. Additionally, the relation $\Omega^{\prime} \subset \subset \Omega$ means that the closure of the set $\Omega^{\prime}$ is contained in $\Omega$.

Theorem 3.2 Let $\chi$ satisfy the Schrödinger equation

$$
i \partial_{t} \chi+\Delta \chi=0
$$

in a bounded open set with smooth boundary $\Omega \subset \mathbb{R}^{n+1}(n \geq 2)$ and take a smaller set $\Omega^{\prime} \subset \subset \Omega$. Suppose that $\chi \in L^{2} H^{s}(\Omega)$ for some $s \in \mathbb{R}$ and that the set $\mathbb{R}^{n} \backslash \Omega_{t}$ is connected for all $t \in \mathbb{R}$. Then, for any $\varepsilon>0$, there is a Schwartz function $\psi_{0} \in \mathcal{S}\left(\mathbb{R}^{n}\right)$ such that 
$\Psi:=e^{i t \Delta} \psi_{0}$ approximates $\chi$ as

$$
\|\chi-\Psi\|_{L^{2} H^{s}\left(\Omega^{\prime}\right)} \leq \varepsilon .
$$

Obviously, $\Psi$ satisfies the Schrödinger equation $i \partial_{t} \Psi+\Delta \Psi=0$ in $\mathbb{R}^{n+1}$.

Remark 3.3 The choice of the norm $L^{2} H^{s}(\Omega)$ in Theorem 3.2 is completely inessential. Instead, one can take $H^{s}(\Omega)$ for any real $s$ or the Hölder norm $C^{s}(\Omega)$ for any $s \geq 0$.

Theorem 3.2 is non-quantitative in the sense that it does not provide any bound ("energetic cost") of the $L^{2}$ norm of $\psi_{0}$ in terms of $\varepsilon$. If the set $\Omega$ where the "local solution" $\chi$ is defined is of cylindrical form

$$
\Omega=D \times \mathbb{R},
$$

where $D \subset \mathbb{R}^{n}$ is a bounded open set with smooth boundary, the above qualitative approximation result can be promoted to a quantitative statement, for details see [6].

\section{Ideas of the proofs}

In this final section we sketch the main ideas to prove the reconnection Theorems 2.1 and 2.3. The complete and detailed arguments can be consulted in $[6,7]$.

\subsection{Sketch of the proof of Theorem 2.1}

Its proof hinges on choosing an initial datum that is the superposition of a finite number of Beltrami fields $\mathcal{W}_{k}$ that oscillate at different large frequencies:

$$
u_{0}=M \mathcal{W}_{0}+\delta_{1} \mathcal{W}_{1}+\cdots+\delta_{n} \mathcal{W}_{n},
$$

Here $\delta_{k}$ are small constants satisfying $\delta_{n} \ll \delta_{n-1} \ll \cdots \ll \delta_{1} \ll 1$. The Beltrami fields $\mathcal{W}_{k}$ satisfy the equation curl $\mathcal{W}_{k}=N_{k} \mathcal{W}_{k}$ in $\mathbb{T}^{3}$ with frequencies $N_{0} \gg N_{1} \gg \cdots \gg N_{n} \gg 1$.

The Beltrami fields $\mathcal{W}_{k}$ for $k$ odd are constructed using the realization theorem proved in [8], which ensures the existence of a Beltrami field $\mathcal{W}_{k}$ with high eigenvalue $N_{k}$ exhibiting a set of vortex lines isotopic to $\mathcal{V}_{k}$; the eigenvalues $N_{k}$ are taken to be odd integers to invoke the inverse localization Theorem 3.1, which is key to prove the aforementioned realization theorem. In contrast, the Beltrami fields $\mathcal{W}_{k}$ for $k$ even are explicit and read in terms of the Cartesian coordinates $\left(x_{1}, x_{2}, x_{3}\right)$ as

$$
\mathcal{W}_{k}:=\left(\sin N_{k} x_{3}, \cos N_{k} x_{3}, 0\right) .
$$

Since the integral curves of these fields lie on the tori $x_{3}=$ const, where $\mathcal{W}_{k}$ is a linear field, it follows that they are all non-contractible.

An essential property of these families is that they are "robustly non-equivalent". Indeed, it can be proved that any small perturbation of a member of the first family (i.e., $k$ odd) exhibits a set of vortex lines isotopic to $\mathcal{V}_{k}$, whereas in the second family (i.e., $k$ even) all the vortex lines are non-contractible.

The global existence of the solution with initial datum $u_{0}$ follows from a suitable stability theorem for the Navier-Stokes equations and the fact that $u_{0}$ is a small perturbation of a Beltrami field. More precisely, the solution to the Navier-Stokes equations with initial datum $v_{0}:=M \mathcal{W}_{0}$ is global and decays exponentially fast as $t$ tends to infinity:

$$
v(x, t)=M \mathcal{W}_{0}(x) e^{-v N_{0}^{2} t} .
$$


We showed in [7] that any initial condition $u_{0}$ that is close enough to $v_{0}$ also gives rise to a global exponentially decaying solution to the Navier-Stokes equations, that is, if $\| u_{0}-$ $v_{0} \|_{C^{r}\left(\mathbb{T}^{3}\right)}<\epsilon$ then

$$
\|u(\cdot, t)-v(\cdot, t)\|_{C^{r}\left(\mathbb{T}^{3}\right)}<C \epsilon e^{\frac{-v t}{2}}
$$

for all $t \geq 0$. For our application we take $r \geq 2$ (to apply the hyperbolic permanence theorem).

Finally, the analysis of the solution to the Navier-Stokes equations with initial datum $u_{0}$, which allows us to establish the existence of vortex reconnection, involves a delicate interplay between the large frequencies $N_{k}$ of the fields and their relative sizes. This ensures that, at time $T_{k}$, the vortex structures of the fluid are related to those of the field $\mathcal{W}_{k}$ in the sense that

$$
u\left(\cdot, T_{k}\right)=c_{k}\left(\mathcal{W}_{k}+\text { small }\right)
$$

for some nonzero constant $c_{k}$. More precisely, a careful choice of the constants $\delta_{k}$ and $N_{k}$, combined with the aforementioned stability theorem, allows us to prove that during the time interval $\left[0, T_{n}\right]$ the evolution of the Navier-Stokes equations with initial condition $u_{0}$ is governed by the heat equation modulo a small error:

$$
u(\cdot, t)=M \mathcal{W}_{0} e^{-v N_{0}^{2} t}+\sum_{k=1}^{n} \delta_{k} \mathcal{W}_{k} e^{-v N_{k}^{2} t}+\text { small }
$$

Therefore, at $t=0$ the field $u_{0}$ is a small perturbation of $M \mathcal{W}_{0}$, and at time $T_{k}$ we have the rescaled field

$$
\begin{aligned}
\delta_{k}^{-1} e^{\nu N_{k}^{2} T_{k}} u\left(\cdot, T_{k}\right)= & \mathcal{W}_{k}+M \delta_{k}^{-1} e^{-v\left(N_{0}^{2}-N_{k}^{2}\right) T_{k}} \mathcal{W}_{0} \\
& +\sum_{1 \leq j \neq k \leq n} \delta_{j} \delta_{k}^{-1} e^{\nu\left(N_{k}^{2}-N_{j}^{2}\right) T_{k}} \mathcal{W}_{j}+\text { small. }
\end{aligned}
$$

All the constants are then carefully chosen so that the second and third summands in this equation are suitably small. The theorem then follows from the properties of the carefully constructed vector fields $\mathcal{W}_{k}$.

Remark 4.1 A scaling argument shows that the frequencies we need to take in the proof of Theorem 2.1 are much larger than $v^{-1 / 2}$. Therefore, there is no hope of making this scenario of vortex reconnection work in the vanishing viscosity limit.

\subsection{Sketch of the proof of Theorem 2.3}

Its proof is based on the construction of a solution to the (linear) Schrödinger equation exhibiting the corresponding reconnection pattern, and then this solution is promoted to a solution of the Gross-Pitaevskii equation that operates in the linear regime (which is achieved by playing with the small rescaling parameter $\eta$ in the statement).

Let $\Sigma \subset \mathbb{R}^{4}$ be a pseudo-Seifert surface connecting the curves $\Gamma_{0}, \Gamma_{1} \subset \mathbb{R}^{3}$ in time $T$. If necessary one can deform the curves $\Gamma_{0}, \Gamma_{1}$ to ensure that $\Sigma$ is in general position with respect to the time axis in the sense that the coordinate $t$ is a Morse function on $\Sigma$.

Using a geometric construction exploiting the triviality of the normal bundle of the pseudoSeifert surface, we constructed in [6] an analytic hypersurface $S$ in $\mathbb{R}^{4}$ that contains $\Sigma$, whose normal direction is never parallel to the time direction. Furthermore, $S$ is diffeomorphic to the product $\Sigma \times(-1,1)$, so one can take a real-valued analytic function $\phi: S \rightarrow \mathbb{R}$ on the 
hypersurface $S$ such that

$$
\phi^{-1}(1)=\Sigma
$$

and its intrinsic gradient $\nabla_{S} \phi$ does not vanish on $\Sigma$.

We consider now the Cauchy problem

$$
i \partial_{t} \chi+\Delta \chi=0,\left.\quad \chi\right|_{S}=\phi, \quad N \cdot \nabla_{x, t} \chi=i,
$$

where $N$ is a unit normal vector to the hypersurface $S$ in $\mathbb{R}^{4}$ and $\nabla_{x, t} \chi$ denotes the spacetime gradient of $\chi$. The hypersurface $S$ is non-characteristic for the Schrödinger equation because $N$ is never parallel to the time direction. Hence the Cauchy-Kovalevskaya theorem ensures that there exists a real analytic solution $\chi$ to the problem (4.2) defined in a neighborhood $V \subset$ $\mathbb{R}^{4}$ of $S$. It can also be shown that $\chi^{-1}(1)=\Sigma$ and that the gradients of the real and imaginary parts of $\chi$ are transverse on $\Sigma$. It is clear, by construction, that $Z_{1-\chi}(t)=\Sigma_{t}$ for all $t$, where we recall that $\Sigma_{t}$ is the intersection of $\Sigma$ with the time $t$ slice. The aforementioned transversality also implies that the set $\chi^{-1}(1)$ is robust under $C^{1}$-small perturbations.

The global approximation Theorem 3.2 guarantees that there exists a Schwartz function $\psi_{0} \in \mathcal{S}\left(\mathbb{R}^{n}\right)$ such that $\Psi:=e^{i t \Delta} \psi_{0}$ approximates the above function $\chi$ as

$$
\|\Psi-\chi\|_{C^{k}(V)}<\varepsilon,
$$

where $k \geq 1$ can be chosen at will.

Let us now consider the rescaled Gross-Pitaevskii equation

$$
i \partial_{t} \tilde{\psi}+\Delta \widetilde{\psi}+\delta\left(1-|\widetilde{\psi}|^{2}\right) \widetilde{\psi}=0
$$

on $\mathbb{R}^{3}$ with initial datum

$$
\widetilde{\psi}(x, 0)=1-\psi_{0}(x),
$$

where $\delta>0$ is a small constant. In view of Duhamel's formula

$$
\widetilde{\psi}(x, t)=1-\Psi(x, t)+i \delta \int_{0}^{t} e^{i(t-s) \Delta}\left(1-|\widetilde{\psi}(x, s)|^{2}\right) \widetilde{\psi}(x, s) d s,
$$

it is standard [6] that, for all small enough $\delta$, there exists a global solution $\widetilde{\psi}$ to this equation with

$$
1-\widetilde{\psi} \in C_{\mathrm{loc}}^{\infty}\left(\mathbb{R}, \mathcal{S}\left(\mathbb{R}^{3}\right)\right)
$$

which is bounded as

$$
\|\Psi-(1-\widetilde{\psi})\|_{C^{k}\left([-T, T] \times \mathbb{R}^{3}\right)} \leq C_{T} \delta
$$

for any $T>0$. The constant $C_{T}$ depends on $T$ and $\psi_{0}$ but not on $\delta$. It then follows from the robust geometric construction above that the zero set $\widetilde{\psi}^{-1}(0) \cap V$ is isotopic to $\Sigma$ (with a diffeomorphism that is $C^{k}$-close to the identity). A further elaboration using Morse theory also allows us to show that this scenario satisfies the $t^{1 / 2}$ law and the change of parity property.

We finally notice that the function

$$
\psi(x, t):=\widetilde{\psi}\left(\delta^{-1 / 2} x, \delta^{-1} t\right)
$$

satisfies the Gross-Pitaevskii equation

$$
i \partial_{t} \psi+\Delta \psi+\left(1-|\psi|^{2}\right) \psi=0, \quad \psi(x, 0)=1-\psi_{0}\left(\delta^{-1 / 2} x\right)
$$

and tends to 1 as $1-\psi \in C_{\mathrm{loc}}^{\infty}\left(\mathbb{R}, \mathcal{S}\left(\mathbb{R}^{3}\right)\right.$ ). Since $\psi$ is just an (anisotropic) rescaling of $\widetilde{\psi}$, we infer that the zero set of $\psi$ is of the form described in the statement of the theorem. 
Acknowledgements A.E. is supported by the ERC Consolidator Grant 862342. D.P.-S. is supported by the grants MTM PID2019-106715GB-C21 and Europa Excelencia EUR2019-103821 from Agencia Estatal de Investigación (AEI). This work is supported in part by the ICMAT-Severo Ochoa grant CEX2019-000904-S and the grant RED2018-102650-T funded by MCIN/AEI/10.13039/501100011033.

Open Access This article is licensed under a Creative Commons Attribution 4.0 International License, which permits use, sharing, adaptation, distribution and reproduction in any medium or format, as long as you give appropriate credit to the original author(s) and the source, provide a link to the Creative Commons licence, and indicate if changes were made. The images or other third party material in this article are included in the article's Creative Commons licence, unless indicated otherwise in a credit line to the material. If material is not included in the article's Creative Commons licence and your intended use is not permitted by statutory regulation or exceeds the permitted use, you will need to obtain permission directly from the copyright holder. To view a copy of this licence, visit http://creativecommons.org/licenses/by/4.0/.

\section{References}

1. Aranson, I.S., Kramer, L.: The world of the complex Ginzburg-Landau equation. Rev. Mod. Phys. 74, 99-143 (2002)

2. Arnold, V.I., Khesin, B.: Topological Methods in Hydrodynamics, 2nd edn. Springer, New York (2021)

3. Bewley, G.P., Paoletti, M.S., Sreenivasan, K.R., Lathrop, D.P.: Characterization of reconnecting vortices in superfluid helium. Proc. Natl. Acad. Sci. 105, 13707-13710 (2008)

4. Browder, F.E.: Approximation by solutions of partial differential equations. Am. J. Math. 84, 134-160 (1962)

5. Constantin, P.: Eulerian-Lagrangian formalism and vortex reconnection. In: Bourgain, J., Kenig, C.E., Klainerman, S. (eds.) Mathematical Aspects of Nonlinear Dispersive Equations, pp. 157-170. Princeton, Princeton University Press (2007)

6. Enciso, A., Peralta-Salas, D.: Approximation theorems for the Schrodinger equation and quantum vortex reconnection. Commun. Math. Phys. 387, 1111-1149 (2021)

7. Enciso, A., Lucà, R., Peralta-Salas, D.: Vortex reconnection in the three dimensional Navier-Stokes equations. Adv. Math. 309, 452-486 (2017)

8. Enciso, A., Peralta-Salas, D., Torres de Lizaur, F.: Knotted structures in high-energy Beltrami fields on the torus and the sphere. Ann. Sci. Éc. Norm. Sup. 50, 995-1016 (2017)

9. Enciso, A., García-Ferrero, M.A., Peralta-Salas, D.: Approximation theorems for parabolic equations and movement of local hot spots. Duke Math. J. 168, 897-939 (2019)

10. Kida, S., Takaoka, M.: Vortex reconnection. Annu. Rev. Fluid Mech. 26, 169-189 (1994)

11. Kleckner, D., Irvine, W.T.M.: Creation and dynamics of knotted vortices. Nat. Phys. 9, 253-258 (2013)

12. Kleckner, D., Kauffman, L.H., Irvine, W.T.M.: How superfluid vortex knots untie. Nat. Phys. 12, 650-655 (2016)

13. Lax, P.D.: A stability theorem for solutions of abstract differential equations, and its application to the study of the local behavior of solutions of elliptic equations. Commun. Pure Appl. Math. 9, 747-766 (1956)

14. Malgrange, B.: Existence et approximation des solutions des équations aux dérivées partielles et des équations de convolution. Ann. Inst. Fourier 6, 271-355 (1955-1956)

15. Monchaux, R., Ravelet, F., Dubrulle, B., Chiffaudel, A., Daviaud, F.: Properties of steady states in turbulent axisymmetric flows. Phys. Rev. Lett. 96, 124502 (2006)

16. Nazarenko, S., West, R.: Analytical solution for nonlinear Schrödinger vortex reconnection. J. Low Temp. Phys. 132, 1-10 (2003)

17. Scheeler, M.W., Kleckner, D., Proment, D., Kindlmann, G.L., Irvine, W.T.M.: Helicity conservation by flow across scales in reconnecting vortex links and knots. Proc. Natl. Acad. Sci. 111, 15350-15355 (2014)

18. Villois, A., Proment, D., Krstulovic, G.: Universal and nonuniversal aspects of vortex reconnections in superfluids. Phys. Rev. Fluids 2, 044701 (2017)

19. Zabusky, N.J., Boratav, O.N., Pelz, R.B., Gao, M., Silver, D., Cooper, S.P.: Emergence of coherent patterns of vortex stretching during reconnection: a scattering paradigm. Phys. Rev. Lett. 67, 2469-2473 (1991)

Publisher's Note Springer Nature remains neutral with regard to jurisdictional claims in published maps and institutional affiliations. 